\title{
Neurodevelopmental Disorder
}

National Cancer Institute

\section{Source}

National Cancer Institute. Neurodevelopmental Disorder. NCI Thesaurus. Code C89338.

A childhood disorder that has a neurological basis and manifests as a developmental disability. 